\title{
Pembrolizumab-Induced Steven-Johnson Syndrome in an NSCLC Patient: A Case Report
}

\author{
Shan Su, ${ }^{1,2}$ Xinxing $\mathrm{Hu}^{2}$, Hongzhong Yang2* \\ ${ }^{1}$ Graduate School of Hunan University of Traditional Chinese Medicine, Changsha, China \\ ${ }^{2}$ Department of Respiratory and Critical Care Medicine, Changsha Central Hospital, Changsha, China \\ Email: *autumnsusan@163.com
}

How to cite this paper: $\mathrm{Su}, \mathrm{S}$., $\mathrm{Hu}, \mathrm{X} . \mathrm{X}$. and Yang, H.Z. (2022) PembrolizumabInduced Steven-Johnson Syndrome in an NSCLC Patient: A Case Report. Health, 14, 57-62.

https://doi.org/10.4236/health.2022.141005

Received: December 15, 2021

Accepted: January 15, 2022

Published: January 18, 2022

Copyright (c) 2022 by author(s) and Scientific Research Publishing Inc. This work is licensed under the Creative Commons Attribution International License (CC BY 4.0).

http://creativecommons.org/licenses/by/4.0/

\section{Open Access}

\begin{abstract}
Background: Immune checkpoints inhibitors (ICIs) are widely used in various therapy of tumors. With the increasing usage of them, immune-related adverse events (irAEs) have been known and become common events, especially in the dermatologic system. However, the rare and severe immune-related cutaneous adverse events (irCAEs) still lack enough knowledge. Case presentation: We described a rare case of Steven-Johnson syndrome (SJS) induced by pembrolizumab in an advanced squamous non-small cell lung cancer (NSCLC) patient. SJS is a rare irCAE that could happen at any time after immunotherapy while this case happened from the 3rd day. The patient had influence-like symptoms and several mucous lesions including oral, eye, and skin. With a gradually severer condition, a stoss therapy of intravenous immunoglobulin (IVIG) had a mild effect. It was a long process and failed to respond to usual dermatologic treatment. Conclusion: We share this case in order to enhance clinicians' ability to early recognition and diagnosis in severe irCAEs. Early recognition and appropriate management are important to evade the termination of immunotherapy. Such severe irCAE should be paid more attention to in clinical medicine when using ICIs.
\end{abstract}

\section{Keywords}

Pembrolizumab, Non-Small Cell Lung Cancer, Immune-Related Cutaneous Adverse Event, Steven-Johnson Syndrome

\section{Introduction}

Immune checkpoints include cytotoxic T-lymphocyte antigen 4 (CTLA-4), programmed cell death 1 (PD1), and programmed cell death ligand 1 (PD-L1). Pembrolizumab is one of the anti-PD1 inhibitors which modulate tumor immunity 
to help longer survival. Many researchers had studied it and it's a standard firstline therapy in advanced metastatic NSCLC and melanoma patients. And of course, there are lots of studies on the most common adverse events, irAE. But unlike usual mild adverse events which are greatly studied, few severe irAE is reported. Here, we present this case of SJS induced by pembrolizumab in order to enhance clinicians' ability to early recognition and diagnosis in such severe cutaneous adverse events (CAEs).

\section{Case Presentation}

A 51-year-old female, presented with a persistent cough, was diagnosed with advanced NSCLC. The pathology of bronchoscopy biopsy confirmed squamous cell lung cancer. Diagnostic imaging data revealed a lesion on the pulmonary artery of mediastinal structures and ipsilateral mediastinal nodes metastasis. According to the American Joint Committee on Cancer (AJCC) $8^{\text {th }}$ edition, the clinical stage was cT4N2M0 III B which was inoperable so that palliative chemotherapy and immunotherapy were recommended. She was admitted to our hospital and was treated with the first cycle of paclitaxel-carboplatin (TC) plus pembrolizumab in 2021.10.22. The accurate dose is as follows: paclitaxel $240 \mathrm{mg}$ Day 1, carboplatin $800 \mathrm{mg}$ Dayl, pembrolizumab $200 \mathrm{mg}$ Day 1. During the implementation of intravenous drip, the therapy above was supplemented by conventional omeprazole, granisetron, fosaprepitant, dexamethasone, diphenhydramine, frusemide, hydratization preventing adverse effects. On the $3^{\text {rd }}$ day, the patient had a fever with a temperature of $38.2^{\circ} \mathrm{C}$. On the $6^{\text {th }}$ day, she occurred sore throat, ophthalmia, oral mucositis, and skin rashes with pruritus. On the $8^{\text {th }}$ day, the symptoms above became more severe. Physical examination showed that macule, maculopapule, small red papule, excoriation occurred on limbs and trunk with diffused distribution. And small blisters occurred on the mouth, redness on the conjunctiva. There's erosion on oral mucosa around lips with pseudomembrane coverage. Then she received an intravenous push of $5 \mathrm{mg}$ dexamethasone without any improvement. The dermatologist, ophthalmologist and stomatologist suggested using topical corticosteroids, antihistamines, eye drops, gargles. On the $10^{\text {th }}$ day, there were multiple oral blisters with pain and increased skin rashes while ophthalmia was getting better. We use an intravenous push of $5 \mathrm{mg}$ dexamethasone again, intravenous drip of $40 \mathrm{mg}$ methylprednisolone still without any change of skin lesions. On the 11th day, there was a wide area of cutaneous lesions, distributing in the face, neck, back, abdomen, waist, arm, legs. Plenty of mahogany, puce macula, maculopapule varied in size, with soybeansize blisters or dry desquamating crust on their surface. Amplifying the cutaneous lesion, the lesion showed erosion when blisters broke. The dermatologist suggested blisters extraction with iodine disinfection, intravenous calcium gluconate, compound glycyrrhizin, vitamin C, cimetidine, $80 \mathrm{mg}$ methylprednisolone twice a day plus $10 \mathrm{~g}$ intravenous immunoglobulin (IVIG)/day. After this treatment, rashes stopped increasing, blisters gradually vanished, pruritus im- 
proved. However, she suffered from myelosuppression after chemotherapy on the $12^{\text {th }}$ day, rashes and blisters increased. We gave a stoss therapy of $20 \mathrm{~g}$ IVIG which didn't work. And the patient was then transferred to dermatology from another hospital. It's a pity that we couldn't follow up on her course. Interestingly, we still could learn a lot from this case (Figure 1).

\section{Discussion}

Immune checkpoints include cytotoxic T-lymphocyte antigen 4 (CTLA-4), programmed cell death 1 (PD1) and programmed cell death ligand 1 (PD-L1). PD1 expression on neoplastic cells enables tumor cells to evade anti-tumor immunity. Pembrolizumab (Keytruda) is a humanized monoclonal anti-PD1 antibody, one of the immune checkpoint inhibitors (CPIs) that regulate the immune response and have been extensively investigated in numerous malignancies including NSCLC. Based on a better prognosis, USA FDA approved pembrolizumab as treating advanced NSCLC and melanoma. Most patients treated with immune checkpoints occurred immune-related adverse events (irAEs). Grades of irAEs were classified by the Common Terminology Criteria for Adverse Events (CTCAE) version 5.0. While $20 \%$ of patients treated with pembrolizumab occurred adverse events, grade $3 / 4$ toxicities were rarely observed [1]. According to KEYNOTE-407 (Clinical Trials.gov, NCT02775435) and a retrospective study, pembrolizumab combined with chemotherapy showed longer progression-free survival (PFS) and overall survival (OS) in patients with previously untreated metastatic squamous NSCLC. It supports that pembrolizumab plus chemotherapy is a standard first-line treatment in metastatic squamous NSCLC patients [2].

Patients treated with pembrolizumab usually have immune-related adverse events (irAE). The appearance of grade 1 - 2 irAEs probably suggests clinical benefit of longer progression-free survival (PFS) and overall survival (OS) and higher overall response rate(ORR) while grade $\geq 3$ irAEs suggests poor prognosis [3] [4]. IrAE could occur in any organ but only $4 \%-5 \%$ of these patients have grade 3 - 4 irAEs [5], life-threatening AEs. According to KEYNOTE-001, KEYNOTE-010, and drug instructions, $65 \%-71 \%$ of NSCLC patients have all grades AEs, and the most common would be: fatigue, decrease in appetite, rash, diarrhea, hypothyroidism, dyspnea, pneumonitis, infusion reaction, autoimmune hepatitis. We could easily figure that immune-related cutaneous adverse events

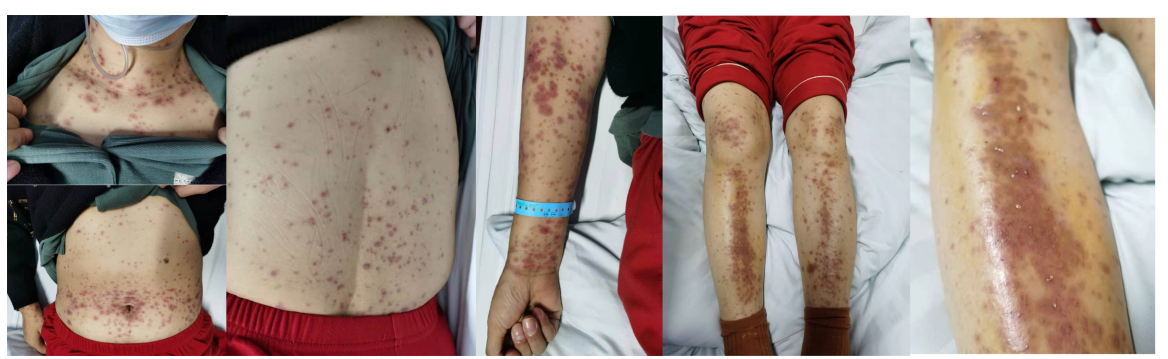

Figure 1. Patient's early cutaneous lesions. 
(irCAEs) are very usual. Classification of irCAEs is still vague and is mostly classified by clinical morphology. Among irCAEs, rashes and pruritus are most common while Stevens-Johnson syndrome (SJS), toxic epidermal necrolysis (TEN), erythema nodosum are rare $(<0.1 \%)$ [1] [6] [7]. Severe cutaneous adverse events (SCARs) include drug reaction with eosinophilia and systemic symptoms (DRESS)/drug-induced hypersensitivity syndrome (DIHS), SJS, TEN, acute generalized exanthematous pustulosis (AGEP) [4]. The onset of SJS, TEN, DRESS could happen at any time, even on the first day after the immunotherapy [8]. SJS was first described in 1922 by Albert Mason Stevens and Frank Chambliss Johnson [9], considered as a delayed hypersensitivity reaction to drugs [10].

SJS/TEN manifest with an influenza-like prodromal phase (malaise, fever). Painful cutaneous and mucous membranes are affected in most patients. The difference between SJS and TEN is in the range of body surface area (BSA). SJS involves $<10 \% \mathrm{BSA}$ while TEN involves $>30 \% \mathrm{BSA}$, the involvement of SJS/TEN is between $10 \%$ and $30 \%$. SJS is rarer [10]. The mechanism of them confers to the activation of $\mathrm{T}$ cells by anti-PD-1 inhibitors resulting in keratinocyte apoptosis, infiltration of CD8+ cells at the dermal-epidermal junction, and CD8+ T cell exocytosis within the epidermis [11]. The histologic feature of SJS/TEN is based on skin necrosis and vasculitis [12]. SJS/TEN commonly had ocular, cutaneous, oral lesions [6] [13]. Many cutaneous eruptions occurred on the arms, trunk, lower extremities [14].

When coming to a SCAR, a skin biopsy may help an accurate diagnosis, and the patients required hospitalization in the dermatologic department or the immediate dermatologic consultation. Supportive care and wound management are the most basic. Topical and systemic steroids, calcineurin inhibitors, immunomodulating and immunosuppressive drugs should be considered [15]. For higher grade SJS patients, they may need: IVIG ( $1-1.5 \mathrm{~g} / \mathrm{kg}$ in single infusion), cyclosporine $(3,5 \mathrm{mg} / \mathrm{kg} /$ day either orally or intravenously divided twice daily dosing), TNF- $\alpha$ (etanercept 25 - $50 \mathrm{mg}$ subcutaneous injections twice a week, infliximab $5 \mathrm{mg} / \mathrm{kg}$ infusion) [5] [6]. After appropriate treatment, clinicians should keep close monitoring of patients on cutaneous lesions [16]. Focusing on our case, this patient has a weight of $70 \mathrm{~kg}$, a height of $163 \mathrm{~cm}$, while we used $20 \mathrm{~g}$ IVIG which may not enough and led to our fail of controlling its progress. And a study showed that higher BMI could be a risk of irAEs [7].

Patients have grade 2 - 3 irCAEs possibly are safe to restart immunotherapy. But grade 4 irCAEs patients should immediately terminate the CPI and never use it. Moreover, clinicians should take close observation of rashes that fail to respond to dermatologic treatment, which may be an early clinical manifestation and a protracted course of SJS. Early recognition and appropriate management of skin toxicity helps decrease the severity of irCAEs, control the eruption of inflammation without interruption of immunotherapy [15] [17].

\section{Conclusion}

We described a rare case of pembrolizumab-associated SJS. In later use of pem- 
brolizumab, clinicians should have the ability to recognize and diagnose severe immune-related cutaneous adverse events while they could manifest as mild skin rashes without responding to usual cutaneous therapy. Early recognition and appropriate management are important to evade the termination of immunotherapy. Further studies are required to evaluate the effect of treatment above for immunotherapy-induced cutaneous severe side-effects such as SJS.

\section{Patient Consent}

The patient consented for images or clinical information relating to her case to be reported in a medical publication.

\section{Competing Interests}

The authors declare that they have no competing interests.

\section{References}

[1] Kwok, G., Yau, T.C., Chiu, J.W., Tse, E. and Kwong, Y.L. (2016) Pembrolizumab (Keytruda). Human Vaccines \& Immunotherapeutics, 12, 2777-2789.

https://doi.org/10.1080/21645515.2016.1199310

[2] Paz-Ares, L., Vicente, D., Tafreshi, A., Robinson, A., Soto Parra, H., Mazières, J., Hermes, B., Cicin, I., Medgyasszay, B., Rodríguez-Cid, J., Okamoto, I., Lee, S., Ramlau, R., Vladimirov, V., Cheng, Y., Deng, X., Zhang, Y., Bas, T., Piperdi, B. and Halmos, B. (2020) A Randomized, Placebo-Controlled Trial of Pembrolizumab plus Chemotherapy in Patients with Metastatic Squamous NSCLC: Protocol-Specified Final Analysis of KEYNOTE-407. Journal of Thoracic Oncology: Official Publication of the International Association for the Study of Lung Cancer, 15, 1657-1669. https://doi.org/10.1016/j.jtho.2020.06.015

[3] Cortellini, A., Chiari, R., Ricciuti, B., Metro, G., Perrone, F., Tiseo, M., Bersanelli, M., Bordi, P., Santini, D., Giusti, R., Grassadonia, A., Di Marino, P., Tinari, N., De Tursi, M., Zoratto, F., Veltri, E., Malorgio, F., Garufi, C., Russano, M., Anesi, C., Buti, S., et al. (2019) Correlations between the Immune-Related Adverse Events Spectrum and Efficacy of Anti-PD1 Immunotherapy in NSCLC Patients. Clinical Lung Cancer, 20, 237-247.e1. https://doi.org/10.1016/j.cllc.2019.02.006

[4] Rose, L.M., DeBerg, H.A., Vishnu, P., Frankel, J.K., Manjunath, A.B., Flores, J. and Aboulafia, D.M. (2021) Incidence of Skin and Respiratory Immune-Related Adverse Events Correlates with Specific Tumor Types in Patients Treated with Checkpoint Inhibitors. Frontiers in Oncology, 10, Article ID: 570752.

https://doi.org/10.3389/fonc.2020.570752

[5] Sun, X., Roudi, R., Dai, T., Chen, S., Fan, B., Li, H., Zhou, Y., Zhou, M., Zhu, B., Yin, C., Li, B. and Li, X. (2019) Immune-Related Adverse Events Associated with Programmed Cell Death Protein-1 and Programmed Cell Death Ligand 1 Inhibitors for Non-Small Cell Lung Cancer: A PRISMA Systematic Review and Meta-Analysis. BMC Cancer, 19, Article No. 558. https://doi.org/10.1186/s12885-019-5701-6

[6] Sosa, A., Lopez Cadena, E., Simon Olive, C., Karachaliou, N. and Rosell, R. (2018) Clinical Assessment of Immune-Related Adverse Events. Therapeutic Advances in Medical Oncology, 10, 1-11. https://doi.org/10.1177/1758835918764628

[7] Eun, Y., Kim, I.Y., Sun, J.M., Lee, J., Cha, H.S., Koh, E.M., Kim, H. and Lee, J. (2019) Risk Factors for Immune-Related Adverse Events Associated with Anti-PD-1 
Pembrolizumab. Scientific Reports, 9, Article No. 14039. https://doi.org/10.1038/s41598-019-50574-6

[8] Geisler, A.N., Phillips, G.S., Barrios, D.M., Wu, J., Leung, D., Moy, A.P., Kern, J.A. and Lacouture, M.E. (2020) Immune Checkpoint Inhibitor-Related Dermatologic Adverse Events. Journal of the American Academy of Dermatology, 83, 1255-1268. https://doi.org/10.1016/j.jaad.2020.03.132

[9] Bakshi, S.S. (2019) Stevens-Johnson Syndrome. Internal and Emergency Medicine, 14, 323-324. https://doi.org/10.1007/s11739-018-1981-0

[10] Lerch, M., Mainetti, C., Terziroli Beretta-Piccoli, B. and Harr, T. (2018) Current Perspectives on Stevens-Johnson Syndrome and Toxic Epidermal Necrolysis. Clinical Reviews in Allergy \& Immunology, 54, 147-176. https://doi.org/10.1007/s12016-017-8654-Z

[11] Muntyanu, A., Netchiporouk, E., Gerstein, W., Gniadecki, R. and Litvinov, I.V. (2021) Cutaneous Immune-Related Adverse Events (irAEs) to Immune Checkpoint Inhibitors: A Dermatology Perspective on Management [Formula: See Text]. Journal of Cutaneous Medicine and Surgery, 25, 59-76. https://doi.org/10.1177/1203475420943260

[12] Kamińska-Winciorek, G., Cybulska-Stopa, B., Lugowska, I., Ziobro, M. and Rutkowski, P. (2019) Principles of Prophylactic and Therapeutic Management of Skin Toxicity during Treatment with Checkpoint Inhibitors. Postepy Dermatologii i Alergologii, 36, 382-391. https://doi.org/10.5114/ada.2018.80272

[13] Yoon, S.Y., Han, J.J., Baek, S.K., Kim, H.J. and Maeng, C.H. (2020) Pembrolizumab-Induced Severe Oral Mucositis in a Patient with Squamous Cell Carcinoma of the Lung: A Case Study. Lung Cancer (Amsterdam, Netherlands), 147, 21-25. https://doi.org/10.1016/j.lungcan.2020.06.033

[14] Shen, J., Chang, J., Mendenhall, M., Cherry, G., Goldman, J.W. and Kulkarni, R.P. (2018) Diverse Cutaneous Adverse Eruptions Caused by Anti-Programmed Cell Death-1 (PD-1) and Anti-Programmed Cell Death Ligand-1 (PD-L1) Immunotherapies: Clinical Features and Management. Therapeutic Advances in Medical Oncology, 10, 1-9. https://doi.org/10.1177/1758834017751634

[15] Apalla, Z., Papageorgiou, C., Lallas, A., Delli, F., Fotiadou, C., Kemanetzi, C. and Lazaridou, E. (2021) Cutaneous Adverse Events of Immune Checkpoint Inhibitors: A Literature Review. Dermatology Practical \& Conceptual, 11, e2021155. https://doi.org/10.5826/dpc.1101a155

[16] Cai, Z.R., Lecours, J., Adam, J.P., Marcil, I., Blais, N., Dallaire, M., Belisle, A. and Mathieu, A. (2020) Toxic Epidermal Necrolysis Associated with Pembrolizumab. Journal of Oncology Pharmacy Practice: Official Publication of the International Society of Oncology Pharmacy Practitioners, 26, 1259-1265. https://doi.org/10.1177/1078155219890659

[17] Coleman, E., Ko, C., Dai, F., Tomayko, M.M., Kluger, H. and Leventhal, J.S. (2019) Inflammatory Eruptions Associated with Immune Checkpoint Inhibitor Therapy: A Single-Institution Retrospective Analysis with Stratification of Reactions by Toxicity and Implications for Management. Journal of the American Academy of Dermatology, 80, 990-997. https://doi.org/10.1016/j.jaad.2018.10.062 